\title{
THE
}

\section{Predictors of Clinical Success Among a National Veterans Affairs Cohort With Methicillin-Resistant Staphylococcus aureus Pneumonia}

\author{
Aisling R. Caffrey \\ University of Rhode Island, aisling_caffrey@uri.edu \\ J. Morrill \\ University of Rhode Island \\ Laura A. Puzniak \\ Kerry L. LaPlante
}

University of Rhode Island, kerrylaplante@uri.edu

Follow this and additional works at: https://digitalcommons.uri.edu/php_facpubs

The University of Rhode Island Faculty have made this article openly available.

Please let us know how Open Access to this research benefits you.

This is a pre-publication author manuscript of the final, published article.

Terms of Use

This article is made available under the terms and conditions applicable towards Open Access Policy Articles, as set forth in our Terms of Use.

\section{Citation/Publisher Attribution}

Caffrey, A. R., Morrill, H. J., Puzniak, L. A., \& LaPlante, K. L. (2014). Predictors of clinical success among a national veterans affairs cohort with methicillin-resistant Staphylococcus aureus pneumonia. Clinical Therapeutics, 35(4), 552-559. doi: 10.1016/j.clinthera.2014.02.013

Available at: http://dx.doi.org/10.1016/j.clinthera.2014.02.013 


\section{Predictors of Clinical Success Among a National Veterans Affairs Cohort with Methicillin- Resistant Staphylococcus aureus Pneumonia}

Aisling R. Caffrey, PhD, MS ${ }^{1,2}$; Haley J. Morrill, PharmD ${ }^{1,2}$; Laura A. Puzniak, PhD, MPH ${ }^{3}$; and Kerry L. LaPlante, PharmD ${ }^{1,2,4}$

${ }^{1}$ Veterans Affairs Medical Center, Infectious Diseases Research Program, Providence, Rhode Island; ${ }^{2}$ University of Rhode Island, College of Pharmacy, Kingston, Rhode Island; ${ }^{3}$ Pfizer Inc, Collegeville, Pennsylvania; ${ }^{4}$ Warren Alpert Medical School of Brown University, Division of Infectious Diseases, Providence, Rhode Island

Address correspondence to: Kerry L. LaPlante, PharmD, Veterans Affairs Medical Center (151), Research Building 35, 830 Chalkstone Avenue, Providence, RI 02908. Tel: 401-273-7100 x2339; Fax: 401-457-3305. E-mail: KerryLaPlante@uri.edu

Running head: Predictors of MRSA Pneumonia Treatment Success

An earlier version of this research was presented at the 52nd Interscience Conference on Antimicrobial Agents and Chemotherapy, September 9-12, 2012, San Francisco, California. 


\section{ABSTRACT}

Background: The treatment of methicillin-resistant Staphylococcus aureus (MRSA) pneumonia is exceedingly complicated, which is concerning because of the high mortality rate associated with the infection. Identification of independent predictors of clinical success can optimize patient care by assisting clinicians in treatment decisions.

Objectives: We sought to identify independent predictors of clinical success in a national Veterans Affairs (VA) cohort of MRSA pneumonia patients.

Methods: A nested case-control study was conducted among a cohort of VA patients with MRSA pneumonia receiving linezolid or vancomycin between January 2002 and September 2010. Cases included those demonstrating clinical success, defined as discharge from the hospital or intensive care unit (ICU) by day 14 after treatment initiation, in the absence of death, therapy change, or intubation by day 14. Controls represented non-success, defined as therapy change, intubation, ICU admission, re-admission, or death between treatment initiation and day 14. The potential predictors assessed included treatment, patient demographics and admission characteristics, previous healthcare and medication exposures, comorbidities, and medical history. Odds ratios (ORs) and 95\% confidence intervals (Cls) were calculated from logistic regression.

Results: Our study included 2442 cases of clinical success and 1290 controls. Demographics varied between the clinical success and non-success groups, including age, race, and region of facility. A current diagnosis of chronic respiratory disease (46\% vs $42 \%$ ) and diagnosis of pneumonia in the year prior to the MRSA pneumonia admission (37\% vs $32 \%$ ) were both more common in the clinical success group. Despite these significant differences, only two predictors of clinical success were identified in our study: previous complication of an implant or graft, including mechanical complications and infections, in the year prior to the MRSA pneumonia admission (OR, 1.55; 95\% CI, 1.17-2.06) and treatment with linezolid (1.53; 1.12-2.10). Predictors of non-success included concomitant urinary tract infection diagnosis 
(OR, 0.82; 95\% Cl, 0.70-0.96), intravenous line $(0.76 ; 0.66-0.89)$, previous coagulopathy $(0.74$; 0.56-0.96), previous amputation procedure $(0.72 ; 0.53-0.98)$, current coagulopathy diagnosis $(0.71 ; 0.53-0.96)$, dialysis $(0.54 ; 0.38-0.76)$, multiple inpatient procedures $(0.53 ; 0.45-0.62)$, inpatient surgery $(0.48 ; 0.41-0.57)$, and previous endocarditis $(0.24 ; 0.07-0.81)$.

Discussion: MRSA pneumonia tends to affect complex patients, and identification of the predictors of clinical success is useful when considering different therapeutic approaches.

Conclusions: In a national cohort of VA patients with MRSA pneumonia, treatment was the only modifiable variable predicting clinical success.

Key words: predictors of clinical success, linezolid, vancomycin, methicillin-resistant Staphylococcus aureus, pneumonia. 


\section{INTRODUCTION}

Pneumonia is the leading cause of infectious disease-related deaths in the United States. ${ }^{1} \mathrm{~A}$ prominent pathogen causing pneumonia in both healthcare and community settings is methicillin-resistant Staphylococcus aureus (MRSA). ${ }^{2}$ In recent years, rates of communityassociated MRSA pneumonia have been steadily increasing, while MRSA was also becoming a leading cause of healthcare-associated pneumonia, including ventilator-associated pneumonia $(\text { VAP) })^{3,4}$

Limited options exist for the treatment of MRSA pneumonia. Vancomycin has been the mainstay of treatment for MRSA infections for years, however rates of treatment failure for pneumonia as high as $40 \%$ have been reported. ${ }^{5}$ Linezolid is a well-studied alternative to vancomycin that has been available for more than 10 years. Several studies have demonstrated clinical equivalence between the 2 drugs, ${ }^{6-9}$ but some data suggest a significant benefit for linezolid compared with vancomycin for the treatment of pneumonia. ${ }^{10-12}$ Currently, both drugs are recommended as first-line options for the treatment of MRSA pneumonia. ${ }^{2}$

In addition to treatment-related factors, host and pathogen characteristics can affect clinical outcomes for patients with MRSA pneumonia. For example, age, underlying comorbidities, severity of illness, multiple lobe involvement, and need for intensive care unit (ICU) admission have been associated with poor clinical outcomes. ${ }^{9-11,13,14}$ There are multiple differences among MRSA strains on a molecular level, including staphylococcal cassette chromosome mec (SCCmec) type, presence of the toxin Panton-Valentine leukocidin, and minimum inhibitory concentration, which can affect clinical outcomes. ${ }^{14-16}$

MRSA pneumonia is a complex disease associated with significant morbidity and mortality. Despite the large public health impact of MRSA pneumonia, there are limited published data examining clinical predictors of success or failure. Identification of independent predictors of clinical success could optimize patient care by assisting clinicians in treatment 
decisions. It was therefore the aim of this study to identify independent predictors of clinical success among a national Veterans Affairs (VA) cohort of MRSA pneumonia patients.

\section{METHODS}

The study design and methods were defined a priori in the study protocol, which was reviewed and approved by the Institutional Review Board and Research and Development Committee of the Providence Veterans Affairs Medical Center.

\section{Data Sources}

The Veterans Health Administration has utilized an electronic medical record system since 1999. Our study included national standardized databases capturing patient care: International Classification of Diseases, 9th Revision (ICD-9) diagnostic and procedure codes, pharmacy records for prescriptions, laboratory tests, select laboratory results, mortality, and patient vital signs. ${ }^{17,18}$

\section{Patient Population and Study Design}

Our nested case-control study was conducted among an original cohort of patients admitted to VA hospitals between January 1, 2002, and September 30, 2010, with an ICD-9 code for MRSA (038.12, 041.12, 482.42, V09.0) and pneumonia (482.40-482.42, 482.49, 482.89, 482.9, 484.8, 485-486, 510.0, 510.9, 513.0-513.1). ${ }^{19}$ Patients aged 18 years or older were selected for inclusion if they were initiated on linezolid or vancomycin during the admission, in the absence of linezolid or vancomycin therapy in the 7 days prior to the hospitalization, with at least 3 days of therapy dosed per hospital protocol. Patients were excluded if they died or were discharged within 3 days of treatment initiation, were initiated on vancomycin or linezolid in the nursing home before their hospital admission, or were exposed to 
more than 2 consecutive days of anti-MRSA antibiotics in the 3 days before treatment initiation or during treatment with linezolid or vancomycin. Only the first admission within the study period meeting all inclusion and exclusion criteria was included.

To identify independent predictors of clinical success among our MRSA pneumonia cohort, we identified cases of clinical success and a non-success control group. Clinical success was defined as discharge from the hospital and/or from the ICU by day 14 after treatment initiation, in the absence of therapy change, intubation, admission to the ICU, re-admission, and/or death by day $14 .{ }^{19}$ Non-success was defined as therapy change, intubation, ICU admission, discharge and readmission, or death between treatment initiation and day $14 .{ }^{19}$ Day 14 was chosen to replicate the average end-of-treatment time frame from existing linezolid and vancomycin clinical trials..$^{6,7,12}$ Our definition of clinical success was defined a priori based on recently published clinical trials utilizing clinical outcomes available from our data source., 6,12

\section{Independent Predictors}

The potential predictors assessed included treatment (linezolid or vancomycin), patient demographics and admission characteristics, comorbidities and medical history, vital signs and laboratory results, previous surgeries and procedures, previous healthcare exposures, and previous medication exposures. ${ }^{19}$ To identify comorbidities and medical history, including sitespecific and organism-specific infections in the year before admission, ICD-9 diagnosis and procedure codes from inpatient and outpatient records were utilized. ${ }^{18}$ Surgeries, procedures, and radiologic examinations during the admission were identified using ICD-9 diagnosis and procedure codes. Hospital admissions, surgeries, and nursing home or extended-care stays were evaluated from 5 non-mutually exclusive time periods ( 30 days, 60 days, 90 days, 180 days, 365 days) to identify previous healthcare exposures. Previous antibiotic and immunosuppressant exposures during the 90 days before admission were identified from inpatient and outpatient prescription records. 


\section{Statistical Analysis}

Between-group differences were assessed using a $x^{2}$ or Fisher's exact test for categorical covariates and the $t$-test or Wilcoxon rank-sum test for continuous variables, as appropriate. An unconditional logistic regression model was developed to identify independent predictors associated with clinical success using a manual backward, non-computer-generated, elimination approach. ${ }^{20}$ We report unadjusted and multivariable adjusted odds ratios (ORs) for each predictor, including their respective $95 \%$ confidence intervals (Cls). All analyses were performed using SAS (SAS Institute Inc., Cary, NC, Version 9.1.3).

\section{RESULTS}

Our study included 2442 cases of clinical success and 1290 non-success controls. There were significant differences in demographics between the clinical success and non-success groups, including age and race (Table I). Patients in the clinical success group were older than patients in the non-success group (mean age $69.5 \pm 12.6$ years vs $68.5 \pm 12.0$ years, $P<0.05$ ). White patients made up a larger proportion of the clinical success group than the non-success group (81.7\% vs $79.1 \%, P<0.05)$. Several significant differences in comorbidities were also observed between groups, including less frequent peripheral vascular disease, coagulopathy, and dialysis and more frequent chronic respiratory disease among patients experiencing clinical success. In addition, patients with clinical success were less likely to have an intravenous line or current complication of an implant or graft and were less likely to be in a surgical unit at treatment initiation.

Treatment with linezolid was more common in the clinical success group ( $6.8 \%$ vs $5.0 \%$, $P<0.05)$, as shown in Table II. Other differences between cases and controls included shorter length of therapy and a larger proportion of subjects with pneumonia infections or previous implant or graft in the last year among those with clinical success. 
Statistically significant predictors of clinical success, as compared with non-success, are shown in Table III. In the adjusted logistic regression model, 2 independent predictors of clinical success were identified: previous complication of an implant or graft, including mechanical complications and infections, in the year prior to the MRSA pneumonia-related admission (adjusted OR, 1.55; 95\% Cl, 1.17-2.06) and treatment with linezolid (1.53; 1.12-2.10). Predictors with Odds Ratios $<1$, and as such associated with non-success, included concomitant urinary tract infection diagnosis (adjusted $\mathrm{OR}, 0.82 ; 95 \% \mathrm{Cl}, 0.70-0.96$ ), intravenous line during the current MRSA pneumonia admission $(0.76 ; 0.66-0.89)$, coagulopathy in the year prior to the MRSA pneumonia admission $(0.74 ; 0.56-0.96)$, previous amputation procedure $(0.72 ; 0.53-0.98)$, current coagulopathy diagnosis $(0.71 ; 0.53-0.96)$, current dialysis $(0.54 ; 0.38-0.76), 3$ or more inpatient procedures $(0.53 ; 0.45-0.62)$ or inpatient surgery $(0.48 ; 0.41-0.57)$ during the current MRSA pneumonia admission, and previous endocarditis $(0.24 ; 0.07-0.81)$.

\section{DISCUSSION}

In our study, the only modifiable variable predicting clinical success among patients with MRSA pneumonia was linezolid treatment. Clinical success was 1.53 times more likely among patients treated with linezolid compared with those treated with vancomycin. Two earlier retrospective post hoc studies demonstrated, in subgroup analyses, that treatment with linezolid (vs vancomycin) was an independent predictor of clinical cure for MRSA pneumonia (OR, 3.3; 95\% CI, 1.3-8.3, $P=0.01)$ and MRSA VAP $(\mathrm{OR}, 20.0 ; 95 \% \mathrm{Cl}, 4.3-92.0, P<0.001) .{ }^{10,11}$ Limitations of these studies include their post hoc design, subgroup analyses, and lack of vancomycin dose optimization.

Several studies have evaluated the comparative clinical efficacy of linezolid and vancomycin with conflicting results. In contrast to our findings, numerous randomized trials have demonstrated no difference in clinical cure rates for the treatment of nosocomial pneumonia 
when comparing linezolid and vancomycin..$^{6-9,21}$ Moreover, in 2 meta-analyses, linezolid treatment for nosocomial pneumonia was not associated with clinical superiority over glycopeptides (vancomycin or teicoplanin). ${ }^{22,23}$

The few trials that support our finding of more frequent clinical success associated with linezolid treatment have been criticized for methodological and statistical flaws. ${ }^{11,12,24-31}$ One of these studies, a recent randomized controlled trial, demonstrated higher clinical success associated with linezolid versus vancomycin in the treatment of MRSA pneumonia. ${ }^{12}$ In this study, $57.6 \%(95 / 165)$ of linezolid patients and $46.6 \%(81 / 174)$ of vancomycin patients achieved clinical success $(95 \% \mathrm{Cl}$ for the difference, $0.5 \%-21.6 \%, P=0.042)$. However, questions regarding vancomycin dose optimization remain, since median trough levels of vancomycin fell below the Infectious Diseases Society of America's recommendations in that trial. ${ }^{2}$ Several retrospective studies have developed algorithms to evaluate clinical success or failure related to MRSA pneumonia. ${ }^{32-34}$ Our study further adds to this developing literature by assessing a large national cohort of patient with MRSA pneumonia using objective clinical outcomes.

It is unclear why a previous complication of an implant or graft was associated with clinical success in our study. Complications included a range of conditions, such as mechanical complications, infections, or other non-specified complications of medical devices, implants, or grafts. ${ }^{18}$ One possible explanation is that this specific medical history could have prompted more aggressive measures to improve the likelihood of clinical success. If this were the case, our findings suggest that MRSA pneumonia patients with previous endocarditis, coagulopathy, and amputation procedures in the year prior to admission may also benefit from additional efforts to improve clinical success.

There are multiple limitations to our study. First, it should be noted that our definition of clinical success varies from those of the aforementioned clinical trials, which affects our ability to make direct comparisons. However, our definition was determined by objective criteria of clinical outcomes rather than clinical judgment as used in those trials. Other limitations include our 
retrospective design and use of diagnosis codes to identify MRSA pneumonia. There may be variations in coding practices across institutions, and the MRSA coding can be discordant with culture-confirmed MRSA. ${ }^{35}$ Furthermore, we were unable to molecularly classify the MRSA isolates; molecular differences in MRSA strains have been shown to affect clinical results. ${ }^{14-16}$ Susceptibility data and trough data were not available, and therefore it is not known if subjects reached therapeutic levels of the treatment agent.

In summary, clinical success in our study was 53\% higher among MRSA pneumonia patients treated with linezolid compared with those treated with vancomycin. Previous complication of an implant or graft in the year prior to the MRSA pneumonia-related admission was also a predictor of clinical success. In addition, concomitant urinary tract infection, intravenous line, previous coagulopathy, previous amputation procedure, current coagulopathy, dialysis, 3 or more inpatient procedures, inpatient surgery, and previous endocarditis were associated with lower odds of clinical success. This study helps to identify patients who may have additional complexities owing to comorbid conditions or medical history and who may benefit from alterations in treatment regimen or require additional attention. Treatment was the only modifiable predictor of clinical success in our study. These findings, based on existing national health data, support the results of previous studies and can assist clinicians in managing patients with MRSA pneumonia.

\section{ACKNOWLEDGMENTS}

Editorial support was provided by Lisa Baker of UBC Scientific Solutions and was funded by Pfizer Inc.

\section{CONFLICTS OF INTEREST}

The views expressed are those of the authors and do not necessarily reflect the position or policy of the United States Department of Veterans Affairs. This material is based upon work 
supported, in part, by the Office of Research and Development, Department of Veterans Affairs. All data collection, extraction, and analyses were carried out by the Department of Veterans Affairs study team. This study was sponsored, in part, by Pfizer Inc. A.R.C. has received research funding from Pfizer Inc. H.J.M. has no conflicts to disclose. L.A.P. is an employee and shareholder of Pfizer Inc. K.L.L. has received research funding or acted as an advisor, speaker, or consultant for Astellas, Cubist, Forest, and Pfizer Inc. 


\section{REFERENCES}

1. Centers for Disease Control and Prevention. Leading causes of death. http://www.cdc.gov/nchs/fastats/lcod.htm. Accessed March 2013.

2. Liu C, Bayer A, Cosgrove SE, et al. Clinical practice guidelines by the Infectious Diseases Society of America for the treatment of methicillin-resistant Staphylococcus aureus infections in adults and children: executive summary. Clin Infect Dis. Feb 1 2011;52(3):285-292 .

3. Kollef MH, Shorr A, Tabak YP, Gupta V, Liu LZ, Johannes RS. Epidemiology and outcomes of health-care-associated pneumonia: results from a large US database of culture-positive pneumonia. Chest. Dec 2005;128(6):3854-3862.

4. David MZ, Daum RS. Community-associated methicillin-resistant Staphylococcus aureus: epidemiology and clinical consequences of an emerging epidemic. Clin Microbiol Rev. Jul 2010;23(3):616-687.

5. Moise PA, Schentag JJ. Vancomycin treatment failures in Staphylococcus aureus lower respiratory tract infections. Int J Antimicrob Agents. Nov 2000;16(Suppl 1):S31-S34.

6. Rubinstein E, Cammarata S, Oliphant T, Wunderink R. Linezolid (PNU-100766) versus vancomycin in the treatment of hospitalized patients with nosocomial pneumonia: a randomized, double-blind, multicenter study. Clin Infect Dis. Feb 1 2001;32(3):402-412.

7. Stevens DL, Herr D, Lampiris H, Hunt JL, Batts DH, Hafkin B. Linezolid versus vancomycin for the treatment of methicillin-resistant Staphylococcus aureus infections. Clin Infect Dis. Jun 1 2002;34(11):1481-1490.

8. Kohno S, Yamaguchi K, Aikawa N, et al. Linezolid versus vancomycin for the treatment of infections caused by methicillin-resistant Staphylococcus aureus in Japan. $J$ Antimicrob Chemother. Dec 2007;60(6):1361-1369. 
9. Wunderink RG, Cammarata SK, Oliphant $\mathrm{TH}$, Kollef $\mathrm{MH}$. Continuation of a randomized, double-blind, multicenter study of linezolid versus vancomycin in the treatment of patients with nosocomial pneumonia. Clin Ther. Mar 2003;25(3):980-992.

10. Kollef MH, Rello J, Cammarata SK, Croos-Dabrera RV, Wunderink RG. Clinical cure and survival in Gram-positive ventilator-associated pneumonia: retrospective analysis of two double-blind studies comparing linezolid with vancomycin. Intensive Care Med. Mar $2004 ; 30(3): 388-394$

11. Wunderink RG, Rello J, Cammarata SK, Croos-Dabrera RV, Kollef MH. Linezolid vs vancomycin: analysis of two double-blind studies of patients with methicillin-resistant Staphylococcus aureus nosocomial pneumonia. Chest. Nov 2003;124(5):1789-1797.

12. Wunderink RG, Niederman MS, Kollef MH, et al. Linezolid in methicillin-resistant Staphylococcus aureus nosocomial pneumonia: a randomized, controlled study. Clin Infect Dis. Mar 1 2012;54(5):621-629.

13. Combes A, Luyt CE, Fagon JY, Wolff M, Trouillet JL, Chastre J. Early predictors for infection recurrence and death in patients with ventilator-associated pneumonia. Crit Care Med. Jan 2007;35(1):146-154.

14. Haque NZ, Arshad S, Peyrani $P$, et al. Analysis of pathogen and host factors related to clinical outcomes in patients with hospital-acquired pneumonia due to methicillinresistant Staphylococcus aureus. J Clin Microbiol. May 2012;50(5):1640-1644.

15. Lessa FC, Mu Y, Ray SM, et al. Impact of USA300 methicillin-resistant Staphylococcus aureus on clinical outcomes of patients with pneumonia or central line-associated bloodstream infections. Clin Infect Dis. Jul 2012;55(2):232-241.

16. Sharma-Kuinkel BK, Ahn SH, Rude TH, et al. Presence of genes encoding pantonvalentine leukocidin is not the primary determinant of outcome in patients with hospitalacquired pneumonia due to Staphylococcus aureus. J Clin Microbiol. Mar $2012 ; 50(3): 848-856$. 
17. Caffrey AR, LaPlante KL. Changing epidemiology of methicillin-resistant Staphylococcus aureus in the Veterans Affairs Healthcare System, 2002-2009. Infection. Jun 2012;40(3):291-297 .

18. Agency for Healthcare Research and Quality. Clinical Classifications Software (CCS) for ICD-9-CM. Healthcare Cost and Utilization Project (HCUP). Rockville, MD: Agency for Healthcare Research and Quality; 2012.

19. Caffrey AR, Morrill HJ, Puzniak LA, LaPlante KL. Comparative effectiveness of linezolid and vancomycin among a national Veterans Affairs cohort with methicillin-resistant Staphylococcus aureus pneumonia. Pharmacother 2014; in press.

20. Hosmer DW, Lemeshow S. Applied Logistic Regression. 2nd ed. New York, NY: John Wiley \& Sons, Inc; 2000.

21. Kaplan SL, Deville JG, Yogev R, et al. Linezolid versus vancomycin for treatment of resistant Gram-positive infections in children. Pediatr Infect Dis J. Aug 2003;22(8):677686.

22. Kalil AC, Murthy MH, Hermsen ED, Neto FK, Sun J, Rupp ME. Linezolid versus vancomycin or teicoplanin for nosocomial pneumonia: a systematic review and metaanalysis. Crit Care Med. Sep 2010;38(9):1802-1808.

23. Walkey AJ, O'Donnell MR, Wiener RS. Linezolid vs glycopeptide antibiotics for the treatment of suspected methicillin-resistant Staphylococcus aureus nosocomial pneumonia: a meta-analysis of randomized controlled trials. Chest. May 2011;139(5):1148-1155.

24. Bauer TT. Nosocomial pneumonia: therapy is just not good enough. Chest. Nov 2003;124(5):1632-1634.

25. Kalil AC, Puumala SE, Stoner J. Unresolved questions with the use of linezolid vs vancomycin for nosocomial pneumonia. Chest. Jun 2004;125(6):2370-2371. 
26. Powers JH, Ross DB, Lin D, Soreth J. Linezolid and vancomycin for methicillin-resistant Staphylococcus aureus nosocomial pneumonia: the subtleties of subgroup analyses. Chest. Jul 2004;126(1):314-315; author reply 315-316.

27. Powers JH, Lin D, Ross D. FDA evaluation of antimicrobials: subgroup analysis. Chest. Jun 2005;127(6):2298-2299; author reply 2300-2301.

28. Lahey T. Questionable superiority of linezolid for methicillin-resistant Staphylococcus aureus nosocomial pneumonia: watch where you step. Clin Infect Dis. Jul 2012;55(1):159-160.

29. Masuta K, Oba Y, Iwata K. Linezolid versus vancomycin for methicillin-resistant Staphylococcus aureus nosocomial pneumonia: controversy continues. Clin Infect Dis. Jul 2012;55(1):161.

30. Torres A. Antibiotic treatment against methicillin-resistant Staphylococcus aureus hospital- and ventilator-acquired pneumonia: a step forward but the battle continues. Clin Infect Dis. Mar 1 2012;54(5):630-632.

31. Wolff M, Mourvillier B. Linezolid for the treatment of nosocomial pneumonia due to methicillin-resistant Staphylococcus aureus. Clin Infect Dis. Jul 2012;55(1):160-161.

32. Hamilton LA, Wood GC, Magnotti LJ, et al. Treatment of methicillin-resistant staphylococcus aureus ventilator-associated pneumonia with high-dose vancomycin or linezolid. J Trauma Acute Care Surg 2012;72:1478-83.

33 Chan JD, Pham TN, Wong J, et al. Clinical outcomes of linezolid vs vancomycin in methicillin-resistant Staphylococcus aureus ventilator-associated pneumonia: retrospective analysis. J Intensive Care Med 2011;26:385-91.

34. Aston JL, Dortch MJ, Dossett LA, Creech CB, May AK. Risk factors for treatment failure in patients receiving vancomycin for hospital-acquired methicillin-resistant Staphylococcus aureus pneumonia.Surg Infect 2010;11:21-8. 
35. Schweizer ML, Rubin MA. The art of oversimplification: the challenge of measuring hospital-acquired MRSA. Infect Control Hosp Epidemiol. Feb 2012;33(2):122-123. 
Table I. Demographics, hospitalization-related characteristics, and comorbidities by clinical success.

\begin{tabular}{|c|c|c|}
\hline Potential Predictors & $\begin{array}{l}\text { Clinical Success } \\
\quad(n=2442)\end{array}$ & $\begin{array}{c}\text { Clinical Non-success } \\
(n=1290)\end{array}$ \\
\hline Age (years), mean (SD)* & $69.5(12.6)$ & $68.5(12.0)$ \\
\hline Male gender, $\mathrm{n}(\%)$ & 2401 (98.3) & $1263(97.9)$ \\
\hline \multicolumn{3}{|l|}{ Race, $n(\%)^{*}$} \\
\hline White & $1996(81.7)$ & $1021(79.1)$ \\
\hline Other/unknown & 446 (18.3) & $269(20.9)$ \\
\hline \multicolumn{3}{|l|}{ Admission source } \\
\hline Home & $919(37.6)$ & $462(35.8)$ \\
\hline Emergency room & $1071(43.9)$ & $588(45.6)$ \\
\hline Hospital & $150(6.1)$ & $83(6.4)$ \\
\hline Nursing home & $302(12.4)$ & $157(12.2)$ \\
\hline \multicolumn{3}{|l|}{ Unit at treatment initiation* } \\
\hline Intensive care & $1042(42.7)$ & $544(42.2)$ \\
\hline Surgery & $121(5.0)$ & $91(7.1)$ \\
\hline General medicine & $1279(52.4)$ & $655(50.8)$ \\
\hline \multicolumn{3}{|l|}{ Treating specialty } \\
\hline Intensive care & $953(39.0)$ & $519(40.2)$ \\
\hline Surgery & $78(3.2)$ & $54(4.2)$ \\
\hline General medicine & $1411(57.8)$ & $717(55.6)$ \\
\hline \multicolumn{3}{|l|}{ Region of facility* } \\
\hline North & $320(13.1)$ & $169(13.1)$ \\
\hline South & $1056(43.2)$ & $620(48.1)$ \\
\hline Midwest & $512(21.0)$ & $243(18.8)$ \\
\hline West & $554(22.7)$ & $258(20.0)$ \\
\hline $\begin{array}{l}\text { Charlson comorbidity index, median } \\
(\mathrm{IQR})^{*}\end{array}$ & $2(1-4)$ & $2(1-4)$ \\
\hline \multicolumn{3}{|l|}{ Comorbid conditions, n (\%) } \\
\hline Chronic respiratory disease & $1123(46.0)$ & $536(41.6)$ \\
\hline Renal disease & 364 (14.9) & $219(17.0)$ \\
\hline Dialysis ${ }^{*}$ & $68(2.8)$ & $87(6.7)$ \\
\hline Diabetes & $778(31.9)$ & $419(32.5)$ \\
\hline Heart failure & $608(24.9)$ & $334(25.9)$ \\
\hline Coronary heart disease & $596(24.4)$ & $296(22.9)$ \\
\hline Cancer & $430(17.6)$ & $247(19.1)$ \\
\hline Amputation* & $59(2.4)$ & $51(4.0)$ \\
\hline Cerebrovascular disease & $215(8.8)$ & $123(9.5)$ \\
\hline Peripheral vascular disease* & $148(6.1)$ & $111(8.6)$ \\
\hline Moderate or severe liver disease & $43(1.8)$ & $30(2.3)$ \\
\hline HIV/AIDS & $25(1.0)$ & $16(1.2)$ \\
\hline Coagulopathy* & $109(4.5)$ & $93(7.2)$ \\
\hline Peripheral line* & $804(32.9)$ & $622(48.2)$ \\
\hline Complication of implant or graft* & $153(6.3)$ & $127(9.8)$ \\
\hline Urinary tract infection* ${ }^{*}$ & $616(25.2)$ & $377(29.2)$ \\
\hline Bacteremia & 475 (19.5) & $263(20.4)$ \\
\hline Skin infection & 560 (22.9) & $323(25.0)$ \\
\hline
\end{tabular}


$\mathrm{SD}=$ standard deviation; $\mathrm{IQR}=$ interquartile range; $\mathrm{MRSA}=$ methicillin-resistant Staphylococcus aureus.

${ }^{*} P<0.05$. 
Table II. Previous infections, healthcare and medication exposures by clinical success.

\begin{tabular}{|c|c|c|}
\hline Potential Predictors & $\begin{array}{l}\text { Clinical Success } \\
(n=2442), n(\%)\end{array}$ & $\begin{array}{l}\text { Clinical Non-success } \\
\quad(n=1290), n(\%)\end{array}$ \\
\hline \multicolumn{3}{|l|}{ Treatment* $^{\star}$} \\
\hline Linezolid & $167(6.8)$ & $64(5.0)$ \\
\hline Vancomycin & $2275(93.2)$ & $1226(95.0)$ \\
\hline Length of therapy, days, median (IQR)* & $7(5-10)$ & $6(4-12)$ \\
\hline Previous hospitalization last year & $1682(68.9)$ & $866(67.1)$ \\
\hline Previous hospitalization last 90 days & $1256(51.4)$ & $655(50.8)$ \\
\hline Previous hospitalization last 30 days & $929(38.0)$ & $497(38.5)$ \\
\hline Previous surgery last year & $496(20.3)$ & $277(21.5)$ \\
\hline Previous surgery last 90 days & $256(10.5)$ & $146(11.3)$ \\
\hline Previous surgery last 30 days & $117(4.8)$ & $72(5.6)$ \\
\hline Previous procedure last year & $1468(60.1)$ & $778(60.3)$ \\
\hline Previous procedure last 90 days & $983(40.3)$ & $539(41.8)$ \\
\hline Previous procedure last 30 days & $608(24.9)$ & $350(27.1)$ \\
\hline $\begin{array}{l}\text { Previous anti-MRSA antibiotics last } 90 \\
\text { days }\end{array}$ & $1108(45.4)$ & $563(43.6)$ \\
\hline $\begin{array}{l}\text { Previous immunosuppressants last } 90 \\
\text { days }\end{array}$ & $60(2.5)$ & $39(3.0)$ \\
\hline Previous corticosteroids last 90 days & $631(25.8)$ & $311(24.1)$ \\
\hline \multicolumn{3}{|l|}{ Previous infections last year } \\
\hline Pneumonia $^{*} \dagger$ & 910 (37.3) & $418(32.4)$ \\
\hline Skin or subcutaneous tissue infection ${ }^{\dagger}$ & $720(29.5)$ & $403(31.2)$ \\
\hline Bacteremia $^{\dagger}$ & $159(6.5)$ & $77(6.0)$ \\
\hline Urinary tract infection $^{\dagger}$ & $592(24.2)$ & $302(23.4)$ \\
\hline Osteomyelitis $^{\dagger}$ & $86(3.5)$ & $58(4.5)$ \\
\hline MRSA & $290(11.9)$ & $160(12.4)$ \\
\hline Pseudomonas aeruginosa & $120(4.9)$ & $58(4.5)$ \\
\hline $\begin{array}{l}\text { Previous complication of implant or graft } \\
\text { last year* }\end{array}$ & $205(8.4)$ & $81(6.3)$ \\
\hline \multicolumn{3}{|c|}{$\begin{array}{l}\text { IQR = interquartile range; MRSA = methicillin-resistant Staphylococcus aureus. } \\
{ }^{*} P<0.05 \text {. }\end{array}$} \\
\hline
\end{tabular}


Table III. Independent predictors of clinical success.

Predictors

Previous complication

of an implant or graft

Treatment (linezolid vs vancomycin)

Urinary tract infection

Peripheral line

Previous coagulopathy

Previous amputation

procedure

Coagulopathy

Dialysis

Inpatient procedures,

3 or more

Inpatient surgery

Previous endocarditis
Unadjusted Odds Ratio

(95\% Confidence Interval)

$1.37(1.05-1.79)$

$1.41(1.05-1.89)$

$0.82(0.70-0.95)$

$0.53(0.46-0.61)$

$0.72(0.56-0.92)$

$0.71(0.53-0.94)$

$0.60(0.45-0.80)$

$0.40(0.29-0.55)$

$0.40(0.35-0.46)$

$0.38(0.33-0.45)$

$0.23(0.07-0.76)$
Adjusted Odds Ratio (95\% Confidence Interval)

1.55 (1.17-2.06)

$1.53(1.12-2.10)$

$0.82(0.70-0.96)$

$0.76(0.66-0.89)$

$0.74(0.56-0.96)$

$0.72(0.53-0.98)$

$0.71(0.53-0.96)$

$0.54(0.38-0.76)$

$0.53(0.45-0.62)$

$0.48(0.41-0.57)$

$0.24(0.07-0.81)$ 